\title{
Numerical and theoretical study of particle saltation on an obliquely oscillating plate
}

\section{AUTHOR(S):}

Kobayakawa, Murino; Fujimoto, Ayumi; Sakata, Mitsuhiro; Yasuda, Masatoshi; Matsusaka, Shuji

\section{CITATION:}

Kobayakawa, Murino ...[et al]. Numerical and theoretical study of particle saltation on an obliquely oscillating plate. Advanced Powder Technology 2014, 25(6): 1854-1859

\section{ISSUE DATE:}

2014-11

URL:

http://hdl.handle.net/2433/196048

\section{RIGHT:}

(c) 2014 The Society of Powder Technology Japan. NOTICE: this is the author's version of a work that was accepted for publication in Advanced Powder Technology. Changes resulting from the publishing process, such as peer review, editing, corrections, structural formatting, and other quality control mechanisms may not be reflected in this document. Changes may have been made to this work since it was submitted for publication. A definitive version was subsequently published in Advanced Powder Technology, 25(6), 2014 doi:10.1016/i.apt.2014.07.017; This is not the published version. Please cite only the published version.; この論文は出版社版でありません。引用の際には出版社版 をご確認ご利用ください。 


\title{
Numerical and theoretical study of particle saltation on an obliquely oscillating plate
}

Murino Kobayakawa, Ayumi Fujimoto, Mitsuhiro Sakata, Masatoshi Yasuda, Shuji Matsusaka*

Department of Chemical Engineering, Kyoto University, Kyoto 615-8510, Japan

* Corresponding author. E-mail address: matsu@cheme.kyoto-u.ac.jp (S. Matsusaka)

\begin{abstract}
Particle saltation on an obliquely oscillating plate is simulated using a mass-point model that considers gravity, fluid resistance, restitution, and friction. The calculated results are in good agreement with results obtained experimentally for particles with different diameters and restitutions. A large particle with high restitution bounces forward and backward repeatedly, whereas a particle with low restitution only bounces forward and consequently has a high transport velocity. The mechanism for the difference in the motion of the particles can be explained by taking into account the phase angle of the oscillating plate and the impulse during particle collision.
\end{abstract}

Keywords: Oblique oscillation; Particle saltation; Restitution coefficient; Fluid resistance; Impulse

\section{Introduction}

Granular materials subjected to vibration exhibit various unusual behaviors, like convection [1-3], bubbling [4-6], segregation [7-9] and vibration-induced air inflow [10]. Particle fluidization under vibration has numerous applications, such as fluidized beds [11, 12], conveyors [13-17], micro-feeders $[18,19]$ and evaluation methods for powder flowability [20-22].

One-dimensional motion analysis of particles bouncing on an oscillating surface has been investigated extensively because they are among the simplest cases of particle fluidization under vibration [23-32]. Luck and Mehta studied the dynamic evolution of a bouncing particle as a function of the coefficient of restitution [25]. By solving Newton's equations of motion for each particle, Luding et al. [26, 27] found that the transition of a particle column from a fluidized state to a condensed state depends on the vibration acceleration, number of particles, and the coefficient of restitution. However, the effects of particle size on the fluid state are not fully understood. In industry, a vibrating conveyor, i.e., an obliquely oscillating trough inducing two-dimensional motion of a particle bed, is used to transport solid materials. In most theoretical studies of this system, the conveying velocity is calculated by assuming that the particle bed is a perfectly inelastic mass-point, and fluid resistance is neglected. Gallas et al. [13] and Simsek et al. [17] simulated the transport of the particle bed on a vibrating conveyor using a discrete particle model. Although many studies on the dynamics of particles under vibration have been conducted, there have been few reports on the two-dimensional motion of individual particles. In particular, there have been few studies on fine particles, because fine particles exhibit complicated behaviors owing to their adhesive and 
cohesive forces, and fluid resistance.

We previously studied the saltation of different-sized particles, from 0.5 to $500 \mu \mathrm{m}$ in diameter, on an obliquely oscillating plate by analyzing images captured by a high-speed microscope camera to examine the effect of the diameter of a particle on its motion [33]. The experimental results showed that the particles with $D_{p 50}=500 \mu \mathrm{m}$ and $50 \mu \mathrm{m}$ saltated individually on the plate, while particles with $D_{p 50}=0.5$ $\mu \mathrm{m}$ formed agglomerated particles with $D_{a g 50}=150 \mu \mathrm{m}$ and the agglomerated particles bounced only slightly. Furthermore, particles with $D_{p 50}=500 \mu \mathrm{m}$ and $50 \mu \mathrm{m}$ were transported by repeated bounces with motion both forward and backward. The ratio of bounces moving backward to the total bounces decreased with decreasing primary particle diameter. The agglomerated particles, on the other hand, always bounced forward and consequently had the highest transport velocity among these particles of different sizes. The differences in motion of the particles of different sizes can be mainly attributed to restitution and fluid resistance.

In this paper, particle saltation on an obliquely oscillating plate is simulated using a mass-point model that considers gravity, fluid resistance, restitution, and friction. As the first step in understanding the mechanism for the difference in the motion of the particles, we apply the simple model that considers dominant factors. The simulated translational motion of three particles with different diameters and coefficients of restitution are compared with the experimental results for $D_{p 50}=500 \mu \mathrm{m}, 50 \mu \mathrm{m}$, and 0.5 $\mu \mathrm{m}$. To clarify the mechanism for difference in the motion of these particles, we theoretically analyzed the collision probability as a function of the phase angle of the oscillating plate and the impulse exerted on the particle during collision.

\section{Numerical model}

A horizontal substrate is driven by an oblique linear oscillation. The $x$ and $y$ coordinates are defined by setting the $x$-axis and $y$-axis tangential and normal (upward) to the substrate, respectively. The horizontal displacement, $x_{\mathrm{s}}$, and vertical displacement, $y_{\mathrm{s}}$, of the oscillating substrate are given by

$$
\begin{aligned}
& x_{s}=A_{x} \sin \omega t \\
& y_{s}=A_{y} \sin \omega t
\end{aligned}
$$

where $A_{x}$ and $A_{y}$ are the horizontal and vertical amplitudes, respectively, $\omega$ is the angular velocity $(=2 \pi f, f$ is the frequency), and $t$ is the time. When the plate is moving upward and downward, it is also moving forward and backward, respectively, because vibration waves in the two directions are synchronized. The equations of motion for a particle during flight are

$$
\begin{gathered}
m_{p} \frac{d v_{p x}}{d t}=F_{d x} \\
m_{p} \frac{d v_{p y}}{d t}=F_{g}+F_{d y}
\end{gathered}
$$


where $m_{p}$ is the mass of the particle, $v_{p}$ is the particle velocity, $F_{g}$ is the gravity, and $F_{d}$ is the fluid resistance. For large particles, $F_{d}$ is negligible compared to $F_{g}$. For smaller particles, $F_{d}$ is given by

$$
F_{d}=-3 \pi \eta v_{p} D_{p}
$$

where $\eta$ is the fluid viscosity $\left(1.8 \times 10^{-5} \mathrm{~Pa} \cdot \mathrm{s}\right)$ and $D_{p}$ is the particle diameter. The relative vertical velocities between the particle and the substrate before and after a collision are related by the coefficient of restitution, $e$, i.e.,

$$
v_{p y 2}-v_{s y 2}=-e\left(v_{p y 1}-v_{s y 1}\right)
$$

where $v_{s y}$ is the vertical velocity of the substrate. The subscripts " 1 " and "2" represent before and after the collision, respectively. We assumed that the mass of the substrate is much greater than that of the particle, and hence the velocity of the substrate after the collision is equal to that before,

$$
v_{s y 2}=v_{s y 1}
$$

The horizontal component, $J_{x}$, and vertical component, $J_{y}$, of the impulse exerted on the particle during the collision with the substrate are

$$
\begin{aligned}
& J_{x}=m_{p}\left(v_{p x 2}-v_{p x 1}\right) \\
& J_{y}=m_{p}\left(v_{p y 2}-v_{p y 1}\right)
\end{aligned}
$$

In collisions that involve sliding, we assume sliding is described by Coulomb's law of friction, resulting in the horizontal and vertical components of the impulse being related by the coefficient of friction, $\mu$,

$$
\begin{array}{ll}
J_{x}=-\mu J_{y} & \left(v_{p \times 1}>v_{s \times 1}\right) \\
J_{x}=0 & \left(v_{p \times 1}=v_{s \times 1}\right) \\
J_{x}=+\mu J_{y} & \left(v_{p \times 1}<v_{s \times 1}\right)
\end{array}
$$

The horizontal rebound velocity of the particle immediately after collision, $v_{p \times 2}$, is obtained by substituting Eqs. (6)-(9) into Eqs. (10.1)-(10.3):

$$
\begin{array}{ll}
v_{p \times 2}=v_{p \times 1}-\mu\left(v_{s y 1}-v_{p y 1}\right)(e+1) & \left(v_{p \times 1}>v_{s \times 1}\right) \\
v_{p \times 2}=v_{p \times 1} & \left(v_{p \times 1}=v_{s \times 1}\right) \\
v_{p \times 2}=v_{p \times 1}+\mu\left(v_{s y 1}-v_{p y 1}\right)(e+1) & \left(v_{p \times 1}<v_{s \times 1}\right)
\end{array}
$$

Here, it is important to note that the tangential component of the relative velocity cannot reverse its direction during the collision because sliding stops when this component decreases to zero. Therefore, it is physically impossible that $v_{p \times 2}$ calculated from Eq. (11.1) is lower than $v_{s \times 1}$, and that $v_{p \times 2}$ calculated from Eq. (11.3) is higher than $v_{s x 1}$. In the critical state, the following equation is employed:

$$
v_{p \times 2}=v_{s \times 1}
$$

The two-dimensional motion of the particle and the substrate can be calculated using Eqs. (1)-(6), (11) and (12). The simulation conditions match the experimental conditions in the previous study [33], i.e., $A_{x}$ $=A_{y}=35 \mu \mathrm{m}$ and $f=280 \mathrm{~Hz}$ for the oscillating substrate, and the parameters of particles are listed in Table 1. 


\section{Results and discussion}

\subsection{Simulated particle trajectories}

Fig. 1 shows the simulated trajectories of particles A, B, and C bouncing on the oscillating plate. Zero vertical displacement indicates the center of the oscillating surface. The trajectories are drawn assuming zero-radius particles. Circles in this figure denote particle-plate collisions, where the displacements are not always zero. The height and direction of each bounce changed depending on the state of the collision. Fig. 1(a) shows that particle A had three larger bounces moving forward (solid line) and two smaller bounces moving backward (dashed line). The range of bounce height was up to approximately $6 \mathrm{~mm}$ and the horizontal distance of each bounce was in the range $-0.8-1.6 \mathrm{~mm}$. Fig. 1(b) shows that particle B had four larger forward bounces and one smaller backward bounce. The particle reached a height of 1.7 $\mathrm{mm}$, and the horizontal distances were in the range $-0.1-1.0 \mathrm{~mm}$. On comparing Fig. 1 (a) and (b), we find that the smaller particle had smaller bounces with asymmetric trajectories because of the effect of fluid resistance during flight. In Fig. 1(c), particle C always bounced forward, with a maximum height of $0.3 \mathrm{~mm}$ and the horizontal distances were in the range $0.1-0.6 \mathrm{~mm}$. The particle with a low restitution bounced only slightly because of larger energy dissipation during collisions.

\subsection{Comparison between the simulation and experimental results}

The bounce heights and distances distributions obtained from the simulation were compared with experimental results [33]. Fig. 2 shows the cumulative distributions of bounce heights obtained from 200 simulated datasets. The plots indicate previous experimental data for $D_{p 50}=500 \mu \mathrm{m}, 50 \mu \mathrm{m}$ and $0.5 \mu \mathrm{m}$ $\left(D_{a g 50}=150 \mu \mathrm{m}\right)$ [33], and lines indicate the simulation results for particle A, B, and C. The height distributions of particle $\mathrm{A}, \mathrm{B}$, and $\mathrm{C}$ had ranges of $0-8 \mathrm{~mm}, 0-2 \mathrm{~mm}$, and $0-0.3 \mathrm{~mm}$, respectively. The range of particle $B$ was lower than that of particle A because of kinetic energy dissipation as a result of fluid resistance during flight. Particle $\mathrm{C}$ had the lowest heights because of nearly perfect inelastic collisions.

Fig. 3 shows the cumulative distributions of the bounce distances. The distributions of particle A, B, and $\mathrm{C}$ had ranges of $-2-5 \mathrm{~mm},-0.3-1.2 \mathrm{~mm}$, and $0-0.6 \mathrm{~mm}$. The ratios of backward bounces to the total number of bounces for particle A, B and, C are 0.33, 0.21, and 0, respectively. Lower bouncing height decreases horizontal distance due to shorter time intervals between successive collisions.

The simulation results were in reasonably good agreement with the experimental data, thus the numerical model will enable the analysis and better understanding of the particle saltation on the obliquely oscillating plate. To better understand the mechanism of forward and backward bounces, we need to consider the state of the collision, i.e., the direction of motion of the plate, and the impulse during collision. Details are discussed in Section 3.3 and 3.4. 


\subsection{Collision probability depending on the phase angle of the oscillating plate}

When the vertical velocity of the incident particle is high enough, the particle can collide with the oscillating plate at all phase angles. However, if the vertical incident velocity is less than the downward velocity of the moving plate, a collision cannot occur. Thus, a lower incident velocity increases the probability of particle collision when the plate is moving upward [23-25,28,33].

Fig. 4 shows the cumulative distributions of the vertical incident velocity $v_{p y 1}$, which are obtained from 200 simulated datasets. The velocity distributions of particle A, B, and C had ranges of $0-500 \mathrm{~mm} / \mathrm{s}, 0-$ $180 \mathrm{~mm} / \mathrm{s}$, and $0-80 \mathrm{~mm} / \mathrm{s}$, respectively, and median velocities of $180 \mathrm{~mm} / \mathrm{s}, 100 \mathrm{~mm} / \mathrm{s}$, and $50 \mathrm{~mm} / \mathrm{s}$. Lower restitution at collision and larger effect of the fluid resistance during flight decreases the particle velocity.

To quantitatively analyze the effect of the fluid resistance on the motion for each particle, the ratio of the fluid resistance to the gravity was calculated from the median velocity in Fig. 4. Table 2 shows the Particle Reynolds number, the fluid resistance, the gravity and the ratio of the fluid resistance to the gravity. For particle A, the fluid resistance has the negligible effect compared to the gravity. The effect of the resistance acting on particle $\mathrm{B}$ is larger due to the smaller particle diameter. The effect for particle $\mathrm{C}$ is smaller than that for particle B because of the larger particle size and the lower particle velocity.

The collision probability as a function of the phase angle $\varphi$ for a one-dimensional vertical oscillating plate can be theoretically analyzed for some typical cases $[23,24,28,33]$. When the vertical velocity of the incident particle $v_{p y 1}$ is greater than that of the plate moving downward, the probability density function $P(\varphi)$ for the sinusoidally oscillating plate is expressed by the following equation [23, 33]:

$$
P(\varphi)=\frac{1}{2 \pi}\left[1-\left.\left(\frac{A_{y} \omega}{v_{p y 1}}\right) \cos \varphi\right|^{\rceil} \quad(0<\varphi<2 \pi)\right.
$$

For $v_{p y 1}<A_{y} \omega, P(\varphi)$ is expressed as [23, 33]:

$$
\begin{aligned}
& P(\varphi)=\frac{1}{2 \pi}\left[1-\left.\left(\frac{A_{y} \omega}{v_{p y 1}}\right) \cos \varphi\right|_{\rfloor} \quad\left(\varphi_{0}<\varphi<\varphi_{1}\right)\right. \\
& =0 \quad\left(0<\varphi<\varphi_{0}, \varphi_{1}<\varphi<2 \pi\right)
\end{aligned}
$$

where $\varphi_{0}$ and $\varphi_{1}$ are the lower and upper limits of the phase angle range in which a collision can occur. $\varphi_{0}$ and $\varphi_{1}$ are determined by

$$
\begin{gathered}
\cos \left(2 \pi-\varphi_{1}\right)=\frac{v_{p y 1}}{A_{y} \omega} \\
\sin \varphi_{0}+\sin \left(2 \pi-\varphi_{1}\right)=\left\{\varphi_{0}+\left(2 \pi-\varphi_{1}\right)\right\} \frac{v_{p y 1}}{A_{y} \omega}
\end{gathered}
$$

The cumulative distribution function $F(\varphi)$ of the probability density function $P(\varphi)$ is obtained from the integration of Eqs. (13) or (14) in the region in which collision can occur. For $v_{p y 1}>A_{y} \omega, F(\varphi)$ is 
expressed as:

$$
F(\varphi)=\frac{1}{2 \pi}\left[\varphi-\left(\frac{A_{y} \omega}{v_{p y 1}}\right) \sin \varphi\right\rceil_{\rfloor} \quad(0<\varphi<2 \pi)
$$

For $v_{p y 1}<A_{y} \omega, F(\varphi)$ is expressed as:

$$
\begin{array}{rlrl}
F(\varphi) & =0 & & \left(0<\varphi<\varphi_{0}\right) \\
& =\frac{1}{2 \pi}\left[\varphi-\varphi_{0}-\left(\frac{A_{y} \omega}{v_{p y 1}}\right)\left(\sin \varphi-\sin \varphi_{0}\right){ }_{\mid}\right. & & \left(\varphi_{0}<\varphi<\varphi_{1}\right) \\
& =1\left(\varphi_{1}<\varphi<2 \pi\right) &
\end{array}
$$

Fig. 5 (a) shows the vertical displacement of the oscillating plate as a function of the phase angle, and Fig. 5 (b) shows the cumulative distribution functions. The dashed lines indicate results calculated from the theoretical probability model as a parameter of the ratio of $v_{p y 1}$ to $A_{y} \omega$, which were obtained using Eqs. (17) and (18). When $v_{p y 1} \geq A_{y} \omega$, the low vertical incident velocity increases the probability of particle collision when plate is moving upward $(\pi / 2<\varphi<3 \pi / 2)$. When $v_{p y 1}$ is infinity, $F(\varphi)$ is proportional to $\varphi$ because the particle can collide with the plate at a constant probability, irrespective of the velocity of the oscillating plate. When $v_{p y 1}<A_{y} \omega$, the distribution curve is discontinuous because the particle cannot collide with the plate in a particular range of the phase angle.

The solid lines indicate the distributions of the simulation. Particle A and B collided with the oscillating plate at all phase angles, whereas particle $C$ did not collide in the range of $0-3 \pi / 4$ and $7 \pi / 4-2 \pi$. The ratios of the particle collisions with the plate moving downward to the total collisions for particle A, B, and C were $0.33,0.23$, and 0.16 , respectively. We observe that particle $\mathrm{C}$ with a lower incident velocity has a lower collision probability with the plate moving downward. The trend of the simulated results agrees with that of the theoretical ones. Therefore, the ratios of the collision are explained by the theoretical probability model.

\subsection{Impulse exerted on the particle during collision}

Fig. 6 shows the cumulative distributions of the rebound horizontal velocities $v_{p \times 2}$ obtained from 200 simulated datasets. The positive and negative values denote forward and backward bounces, respectively. The rebound velocity distributions of particles A, B, and C had the ranges of $-45-62 \mathrm{~mm} / \mathrm{s},-18-62$ $\mathrm{mm} / \mathrm{s}$, and $0-62 \mathrm{~mm} / \mathrm{s}$. Particles A, B, and C had median rebound velocities of $15 \mathrm{~mm} / \mathrm{s}, 19 \mathrm{~mm} / \mathrm{s}$, and $37 \mathrm{~mm} / \mathrm{s}$. The ratios of the backward bounces to the total bounces for particle A, B, and C are $0.33,0.21$, and 0 , respectively. The ranges of the distributions were within the range of the oscillating plate velocity, i.e. -62 to $62 \mathrm{~mm} / \mathrm{s}$, because the friction force exerted on the particle during collision resisted the relative motion between the particle and plate. The particle with higher incident vertical velocity had wider range of the rebound horizontal velocity because it collided with the oscillating plate at wider phase range, as shown in section 3.3. Details of the difference in the ratios of the backward bounces are discussed below. 
Table 3 summarizes the ratios of the collisions with the plate moving downward $N_{\text {down }}$ to the total number of collisions $N_{\text {total }}$ and the ratios of the backward bounces $N_{\text {back }}$ to the total number of bounces $N_{\text {total }}$. For particle A and B, $N_{\text {down }} / N_{\text {total }}$ is equal to $N_{\text {back }} / N_{\text {total }}$, whereas the values for particle C were significantly different. This feature can be explained as follows: (i) a higher incident velocity increases the vertical component of the impulse, $J_{y}$, exerted on a particle during the collision; (ii) the vertical impulse directly affects the horizontal component of the impulse, $J_{x}$, as seen from Eqs. (10.1) and (10.3), and induces a change in the horizontal velocity of the particle after collision; (iii) for particle C, the impulse exerted on the particle is, however, too small to reverse its direction owing to its low restitution (see Table 1).

Next, we consider the case of a particle collision that can reverse the horizontal direction of the particle. Fig. 7 shows the rebound horizontal velocity $v_{p x 2}$ as a function of the horizontal incident velocity $v_{p x 1}$. This calculation was carried out using Eq. (11.1) with $v_{s x}=v_{s y}=-30 \mathrm{~mm} / \mathrm{s}$ and the median values in Fig. 4 were used for $v_{p y 1}$, showing that particle A, B and C bounce backward where the horizontal incident velocities are less than 29,11 , and $3 \mathrm{~mm} / \mathrm{s}$, respectively.

From the analysis of the impulse in this Section and the analysis of the collision probability in Section 3.3, it is concluded that particle $\mathrm{C}$ rarely bounces backward because the particle has a lower collision probability with the plate moving backward and even if it collide with the plate moving backward, the impulse exerted on the particle is too small to reverse its direction.

\subsection{Analysis of transport velocity}

Fig. 8 shows the cumulative distributions of the average horizontal velocities during each bounce. The plots indicate previous experimental data for $D_{p 50}=500 \mu \mathrm{m}, 50 \mu \mathrm{m}$ and $0.5 \mu \mathrm{m}\left(D_{a g 50}=150 \mu \mathrm{m}\right)$ [33], and lines indicate the simulation results for particle A, B, and C. The trend of the simulated results was in reasonable agreement with that of the experimental data although there is a slight difference for particle C. The horizontal velocity distributions of particle A, B and C had ranges of $-45-62 \mathrm{~mm} / \mathrm{s},-13-35 \mathrm{~mm} / \mathrm{s}$, and $0-62 \mathrm{~mm} / \mathrm{s}$, and median velocities of $15 \mathrm{~mm} / \mathrm{s}, 10 \mathrm{~mm} / \mathrm{s}$, and $33 \mathrm{~mm} / \mathrm{s}$. From these results, it was found that particle $\mathrm{C}$ with lower restitution had the highest transport velocity even though the bounces were small.

Lastly, we compare particle A with particle B. Particle B has a lower ratio of backward bounces and a higher median rebound horizontal velocity (Fig. 6). However, the transport velocity of particle B is lower than that of particle A in Fig. 8. This is because the effect of the fluid resistance exerted on particle B is more than that on particle A. Thus, the transport velocity of particle B is lower.

\section{Conclusions}


The saltation of particles on an obliquely oscillating plate was simulated using a mass-point model that considered gravity, fluid resistance, restitution, and friction. The simulated translational motion for three particles with different diameters and coefficients of restitution was in good agreement with experimental results, proving that a large particle with high restitution was transported by repeated larger bounces and with both forward and backward motion, whereas a particle with a low restitution slightly saltated and only bounced forward. The differences in the motion can be explained as follows: low restitution during a collision and large fluid resistance during flight induce small bounces, and low vertical incident velocities cause a low probability of collision with the plate moving downward, hence low impulses exerted on the particle during collision. As a result, the horizontal direction of the particle with low restitution does not reverse, and consequently the transport velocity becomes higher than that of particles with high restitution.

\section{Acknowledgments}

This work was supported by JSPS KAKENHI Grant Number 25-1710. The authors acknowledge the financial support.

\section{References}

[1] C. Laroche, S. Douady, S. Fauve, Convective flow of granular masses under vertical vibrations, J. Phys. (Paris) 50 (1989) 699-706.

[2] Y-h. Taguchi, New origin of a convective motion: Elastically induced convection in granular materials, Phys. Rev. Lett. 69 (1992) 1367-1370.

[3] J. A. C. Gallas, H. J. Herrmann, S. Sokolowski, Convection cells in vibrating granular media, Phys. Rev. Lett. 69 (1992) 1371-1374.

[4] H. K. Pak, P. R. Behringer, Bubbling in vertically vibrated granular materials, Nature (London) 371 (1994) 231-233.

[5] P. Zamankhan, Bubbles and solid structures in a vibrated bed of granular materials, Physica A 390 (2011) 1402-1416.

[6] P. Zamankhan, Bubbling in vibrated granular films, Phys. Rev. E 83 (2011) 021306.

[7] J. B. Knight, H. M. Jaeger, S. R. Nagel, Vibration-induced size separation in granular media: The convection connection, Phys. Rev. Lett. 70 (1993) 3728-3731.

[8] W. Cooke, S. Warr, J. M. Huntley, R. C. Ball, Particle size segregation in a two-dimensional bed undergoing vertical vibration, Phys. Rev. E 53 (1996) 2812-2822.

[9] A. Kudrolli, Size separation in vibrated granular matter, Rep. Prog. Phys. 67 (2004) 209-247.

[10] S. Matsusaka, M. Kobayakawa, M. Mizutani, M. Imran, M. Yasuda, Bubbling behavior of a fluidized bed of fine particles caused by vibration-induced air inflow, Sci. Rep. 3 (2013) 1190. 
[11] J. R. Wank, S. M. George, A. W. Weimer, Vibro-fluidization of fine boron nitride powder at low pressure. Powder Technol. 121 (2001) 195-204.

[12] C. H. Nam, R. Pfeffer, R. N. Dave, S. Sundaresan, Aerated vibrofluidization of silica nanoparticles, AIChE J. 50 (2004) 1776-1785.

[13] J. A. C. Gallas, H. J. Herrmann, S. Sokolowski, Two-dimensional powder transport on a vibrating belt, J. Phys. II France 2 (1992) 1389-1400.

[14] F. J. C. Rademacher, L. ter Borg, On the theoretical and experimental conveying speed of granular bulk solids on vibratory conveyors, Forsch. Ingenieurwes. 60 (1994) 261-283.

[15] E. M. Sloot, N. P. Kruyt, Theoretical and experimental study of the transport of granular materials by inclined vibratory conveyors, Powder Technol. 87 (1996) 203-210.

[16] R. Grochowski, P. Walzel, M. Rouijaa, C. A. Kruelle, I. Rehberg, Reversing granular flow on a vibratory conveyor, Appl. Phys. Lett. 84 (2004) 1019-1021.

[17] E. Simsek, S. Wirtz, V. Scherer, H. Kruggel-Emden, R. Grochowski, P. Walzel, An experimental and numerical study of transversal dispersion of granular material on a vibrating conveyor, Part. Sci. Technol. 26 (2008) 177-196.

[18] S. Matsusaka, M. Urakawa, H. Masuda, Micro-feeding of fine powders using a capillary tube with ultrasonic vibration, Adv. Powder Technol. 6 (1995) 283-293.

[19] S. Matsusaka, K. Yamamoto, H. Masuda, Micro-feeding of a fine powder using a vibrating capillary tube, Adv. Powder Technol. 7 (1996) 141-151.

[20] Y. Jiang, S. Matsusaka, H. Masuda, Y. Qian, Development of measurement system for powder flowability based on vibrating capillary method, Powder Technol. 188 (2009) 242-247.

[21] K. Ishii, M. Suzuki, T. Segawa, Y. Kihara, M. Yasuda, S. Matsusaka, A vibrating tube method for evaluating flowability of a small amount of sample particles, Adv. Powder Technol. 22 (2011) 522-525.

[22] I. M. Zainuddin, M. Yasuda, T. Horio, S. Matsusaka, Exeperimental study on powder flowability using vibration shear tube method, Part. Part. Syst. Char. 29 (2012) 8-15.

[23] C. C. Feng, K. F. Graff, Impact of a spherical tool against a sonic transmission line, J. Acoust. Soc. Am. 52 (1972) 254-259.

[24] L. A. Wood, K.P. Byrne, Analysis of a random repeated impact process, J. Sound Vib. 78 (1981) 329-345.

[25] J. M. Luck, A. Mehta, Bouncing ball with a finite restitution: Chattering, locking, and chaos, Phys. Rev. E 48 (1993) 3988-3997.

[26] E. Clement, S. Luding, A. Blumen, J. Rajchenbach, J. Duran, Fluidization, condensation and clusterization of a vibrating column of beads, J. Mod. Phys. B 7 (1993) 1807-1827.

[27] S. Luding, E. Clement, A. Blumen, J. Rajchenbach, J. Duran, Studies of columns of beads under external vibrations, Phys. Rev. E 49 (1994) 1634-1646.

[28] S. Warr, J. M. Huntley, Energy input and scaling laws for a single particle vibrating in one dimension, 
Phys. Rev. E 52 (1995) 5596-5601.

[29] E. Falcon, C. Laroche, S. Fauve, C. Coste, Collision of a 1-D column of beads with a wall, Eur. Phys. J. B 5 (1998) 111-131.

[30] J. -C. Germinard, C. Laroche, Energy of a single bead bouncing on a vibrating plate: Experiments and numerical simulations, Phys. Rev. E 68 (2003) 031305.

[31] J. J. Barroso, M. V. Carneiro, E. E. N. Macau, Bouncing ball problem: Stability of the periodic modes, Phys. Rev. E 79 (2009) 026206.

[32] G. Lumay, S. Dorbolo, O. Gerasymov, N. Vandewalle, Experimental study of a vertical column of grains submitted to a series of impulses, Eur. Phys. J. E 36 (2013) 16.

[33] M. Kobayakawa, M. Yasuda, S. Matsusaka, Microscopic analysis of saltation of particles on an obliquely oscillating plate, Adv. Powder Technol. (submitted) 
Table 1

Parameters of particles used in the simulation.

\begin{tabular}{cccc}
\hline Particle & A & B & C \\
\hline Particle diameter $D_{p}(\mu \mathrm{m})$ & 500 & 50 & 150 \\
Particle density $\rho_{p}\left(\mathrm{~kg} / \mathrm{m}^{3}\right)$ & 3900 & 3900 & 930 \\
Coefficient of restitution $e(-)$ & 0.9 & 0.9 & 0.1 \\
Coefficient of friction $\mu(-)$ & 0.1 & 0.1 & 0.2 \\
\hline
\end{tabular}

\section{Table 2}

Particle Reynolds number, fluid resistance, gravity and ratio of fluid resistance to gravity.

\begin{tabular}{cccc}
\hline Particle & A & B & C \\
\hline Particle Reynolds number $R e_{p}(-)$ & 6.0 & 0.27 & 0.50 \\
Fluid resistance $F_{d}(\mathrm{nN})$ & 16 & 0.69 & 1.3 \\
Gravity $F_{g}(\mathrm{nN})$ & 2500 & 2.5 & 16 \\
$F_{d} / F_{g}(-)$ & 0.01 & 0.28 & 0.08 \\
\hline
\end{tabular}

Table 3

Ratios of the plate moving downward to the total collisions and ratios of the backward bounces to the total bounces.

\begin{tabular}{cccc}
\hline Particle & A & B & C \\
\hline$N_{\text {down }} / N_{\text {total }}$ & 0.33 & 0.23 & 0.16 \\
$N_{\text {back }} / N_{\text {total }}$ & 0.33 & 0.21 & 0 \\
\hline
\end{tabular}


Figure contents

Fig. 1. Simulated trajectories for (a) particle A, (b) particle B, and (c) particle C. Zero vertical displacement indicates the center of the oscillation. Solid and dashed lines indicate bounces moving forward and backward, respectively, and circles denote the collision of the particles with the oscillating plate.

Fig. 2. Cumulative distributions of bouncing heights. Each distribution curve consists of 200 simulated datasets. Plot points indicate experimental data for $D_{p 50}=500 \mu \mathrm{m}, 50 \mu \mathrm{m}$ and $0.5 \mu \mathrm{m}\left(D_{a g 50}=150 \mu \mathrm{m}\right)$ [33], and lines indicate simulation results for particle A, B, and C.

Fig. 3. Cumulative distributions of bouncing distances. Each distribution curve consists of 200 simulated datasets. Plot points indicate experimental data from the previous study.

Fig. 4. Cumulative distributions of vertical incident velocities obtained from the simulation.

Fig. 5. (a) Vertical displacement of plate as a function of phase angle. (b) Cumulative distribution functions of particle collision with the oscillating plate. The dashed lines indicate results calculated from the theoretical probability model as a parameter of the ratio of $v_{p y 1}$ to $A_{y} \omega$, which were obtained using Eqs. (17) and (18). The solid lines indicate the distributions from the simulation for particles A, B, and C.

Fig. 6. Cumulative distributions of horizontal rebound velocities obtained from the simulation.

Fig. 7. Horizontal rebound velocity $v_{p x 2}$ as a function of the horizontal incident velocity $v_{p x 1}$ determined by Eq. (11.1) for $v_{s x}=v_{s y}=-30 \mathrm{~mm} / \mathrm{s}$, where the vertical incident velocity $v_{p y 1}$ is the median velocity for each particle in Fig. 4.

Fig. 8. Cumulative distributions of average horizontal velocities during each bounce obtained from simulation. Plot points indicate experimental data from the previous study. 
Fig. 1.

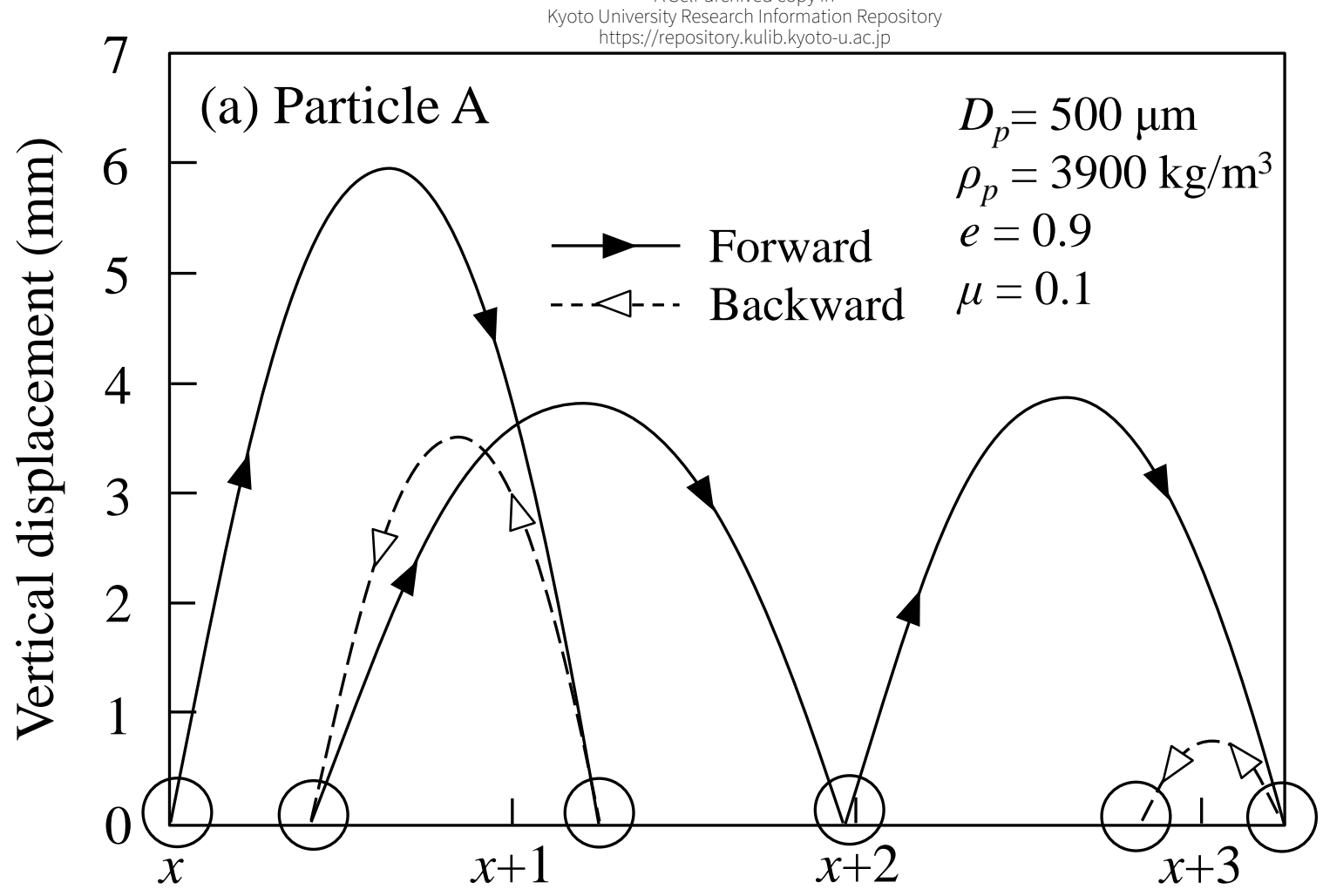

Horizontal displacement (mm)
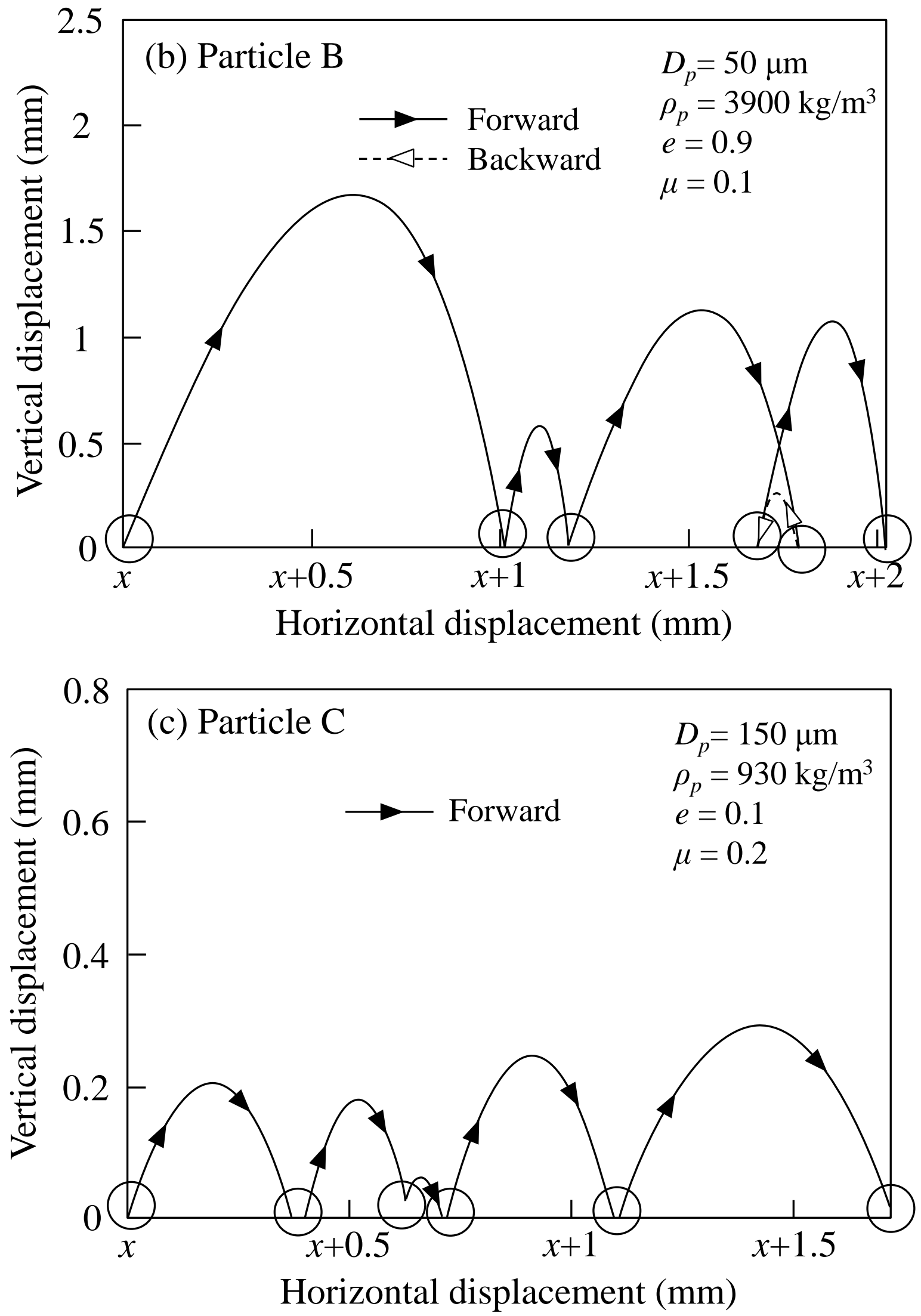
Fig. 2.

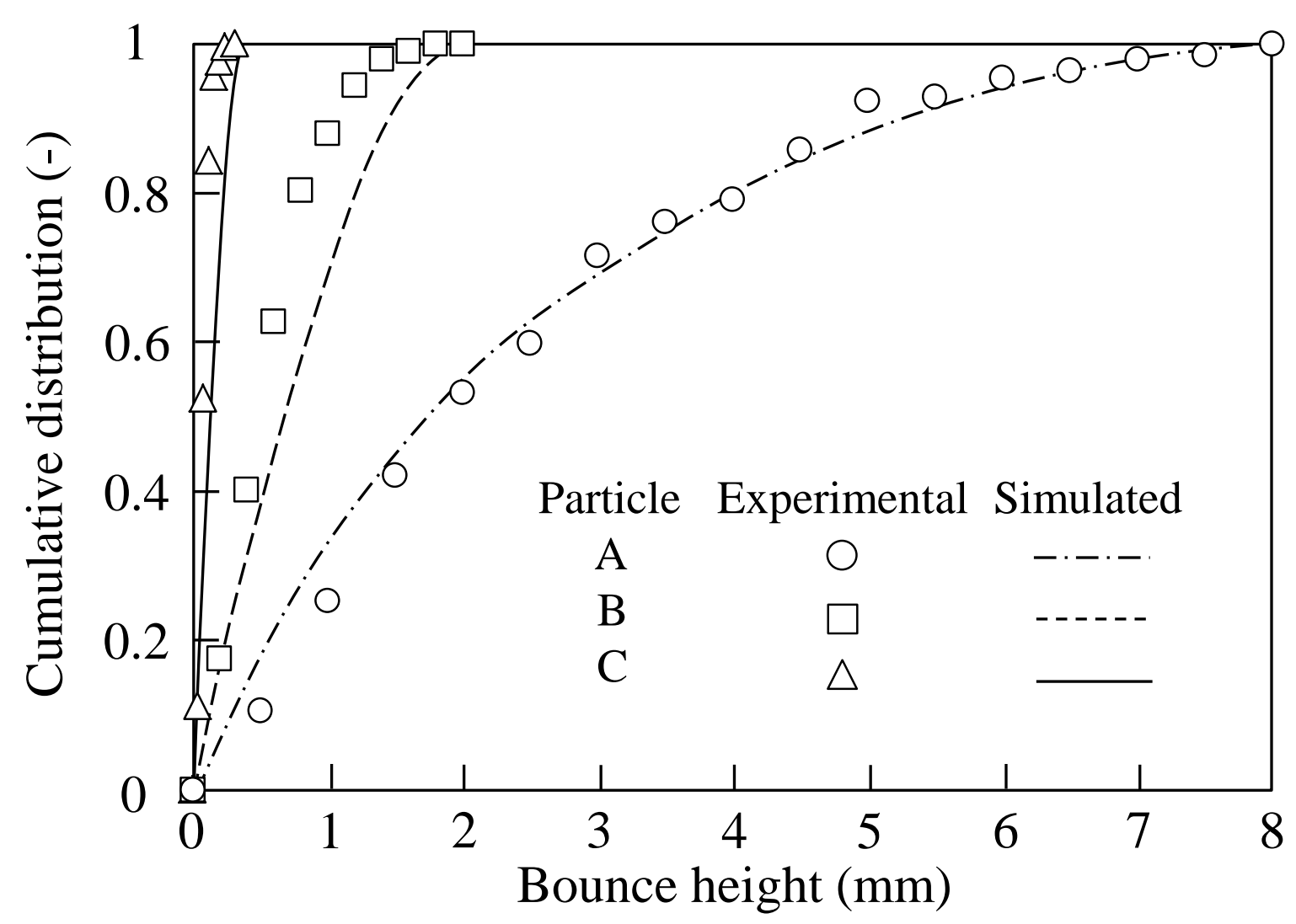


Fig. 3.

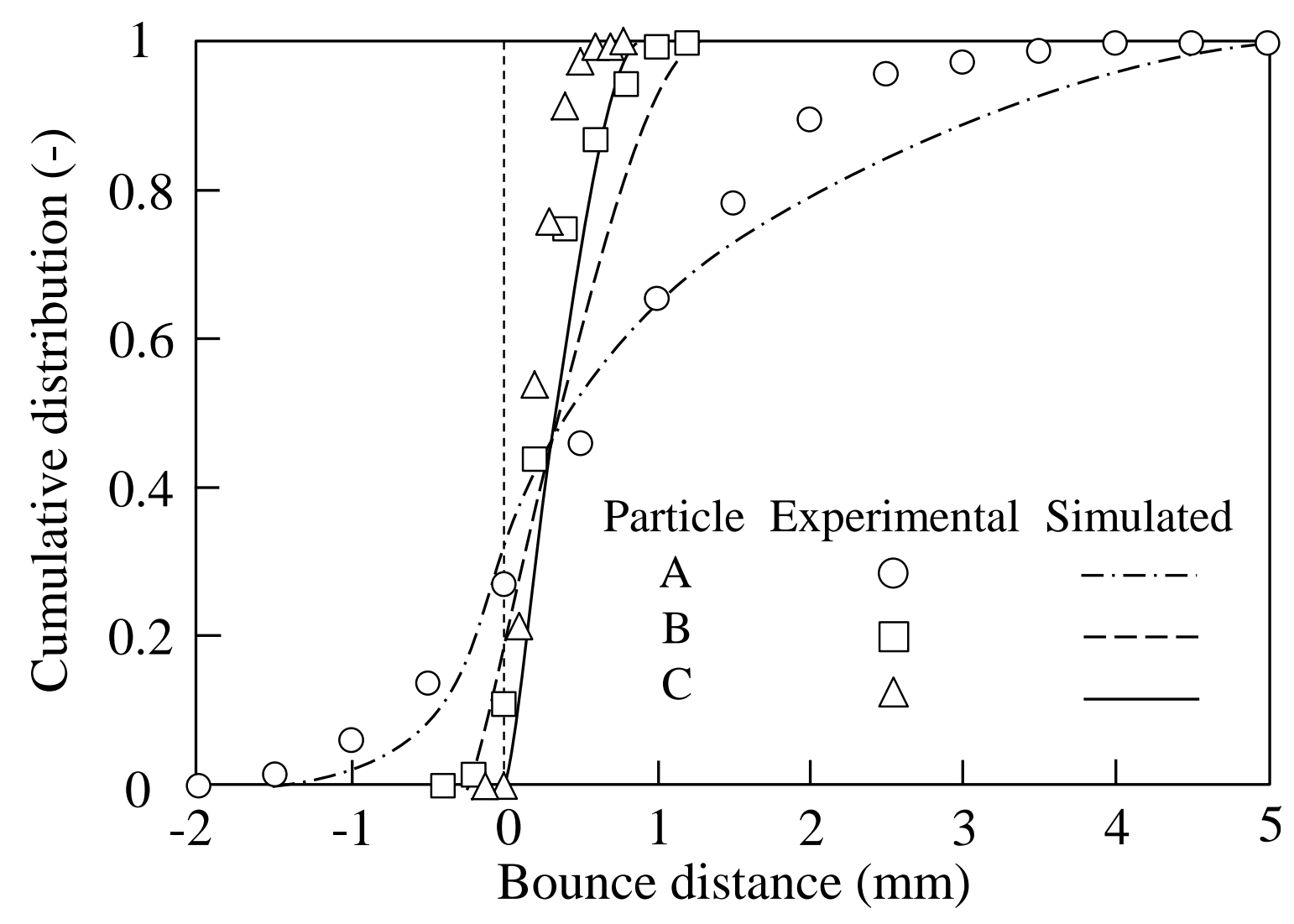


Fig. 4.

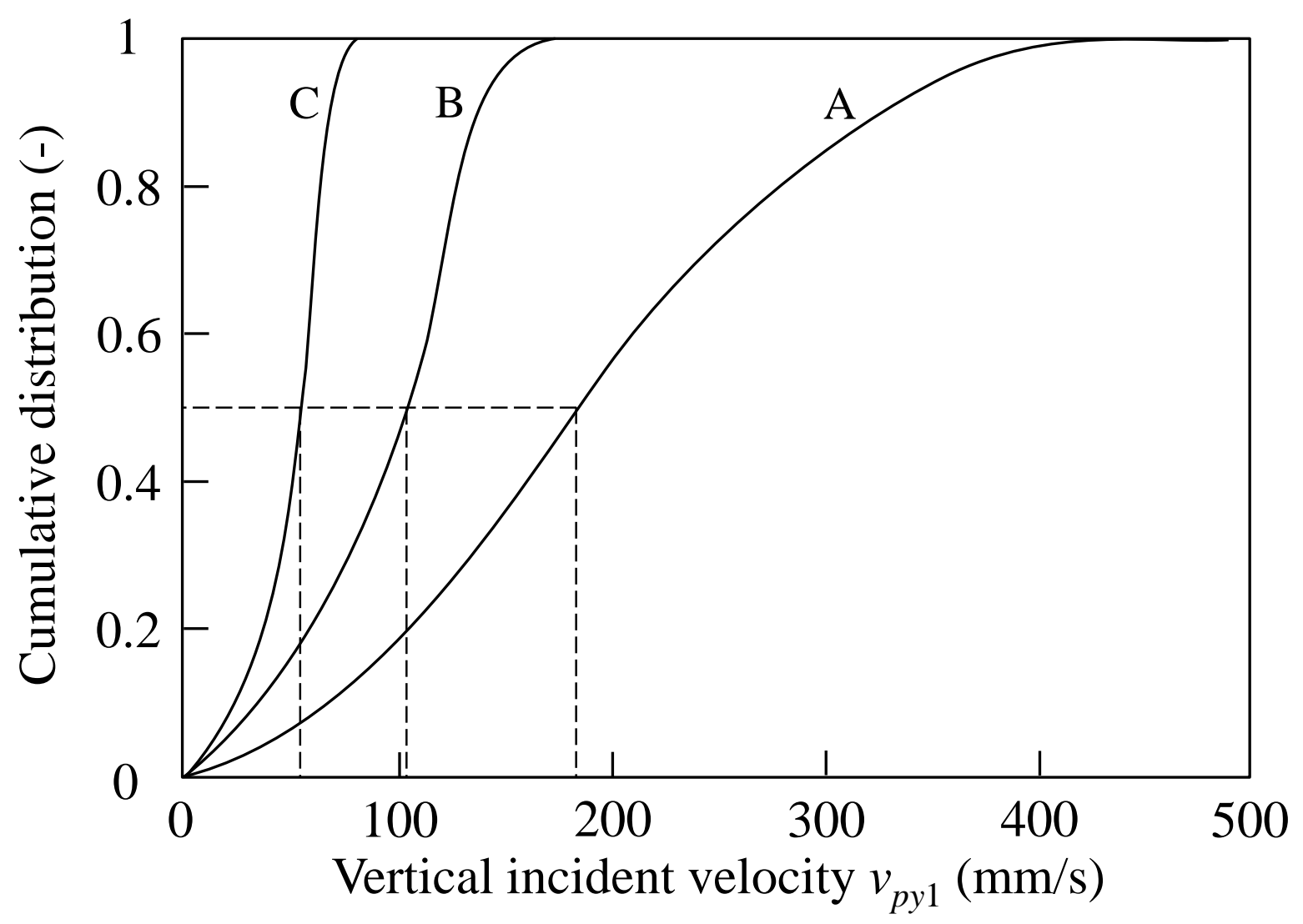


Fig. 5.

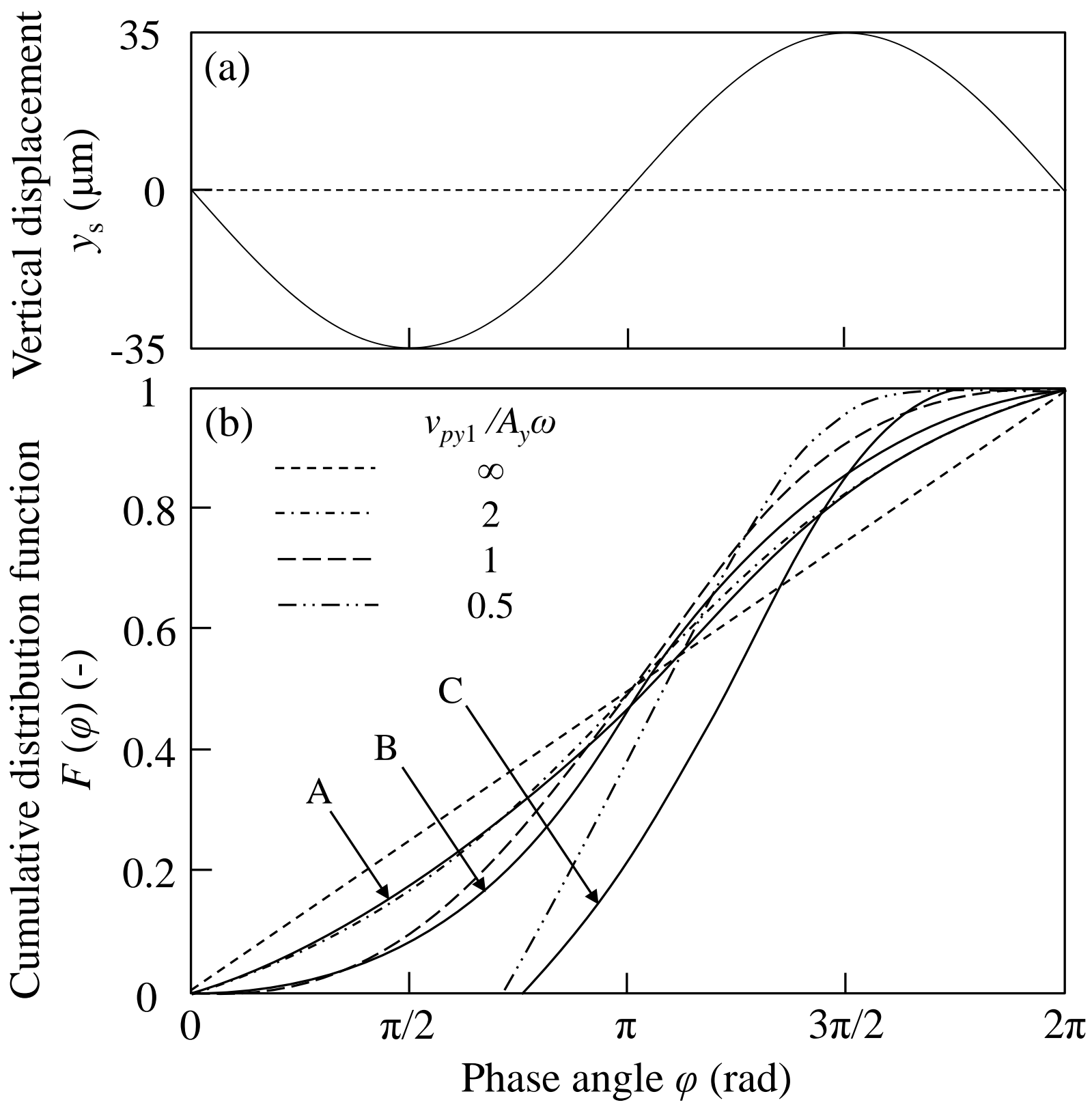


Fig. 6.

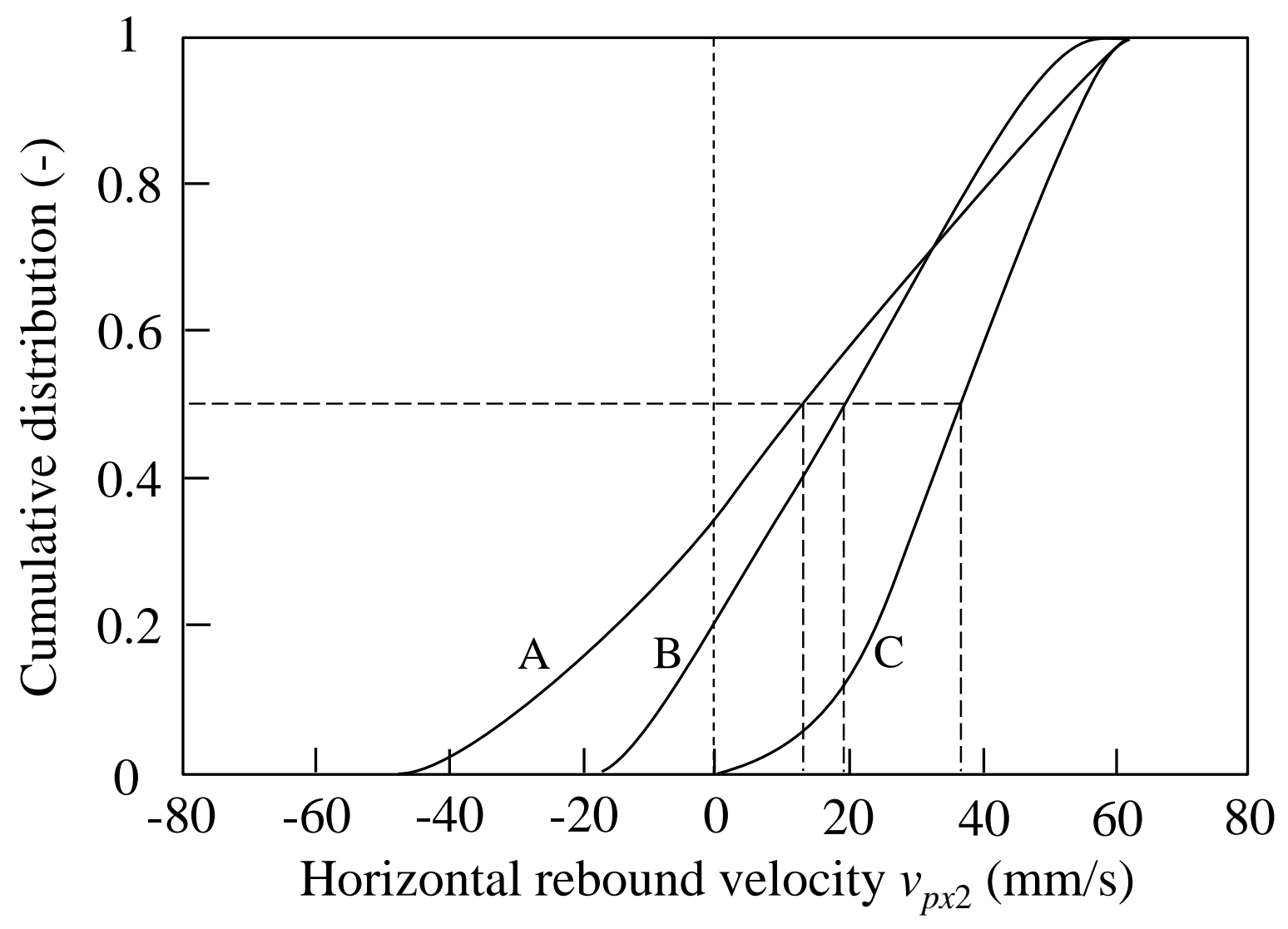


Fig. 7.

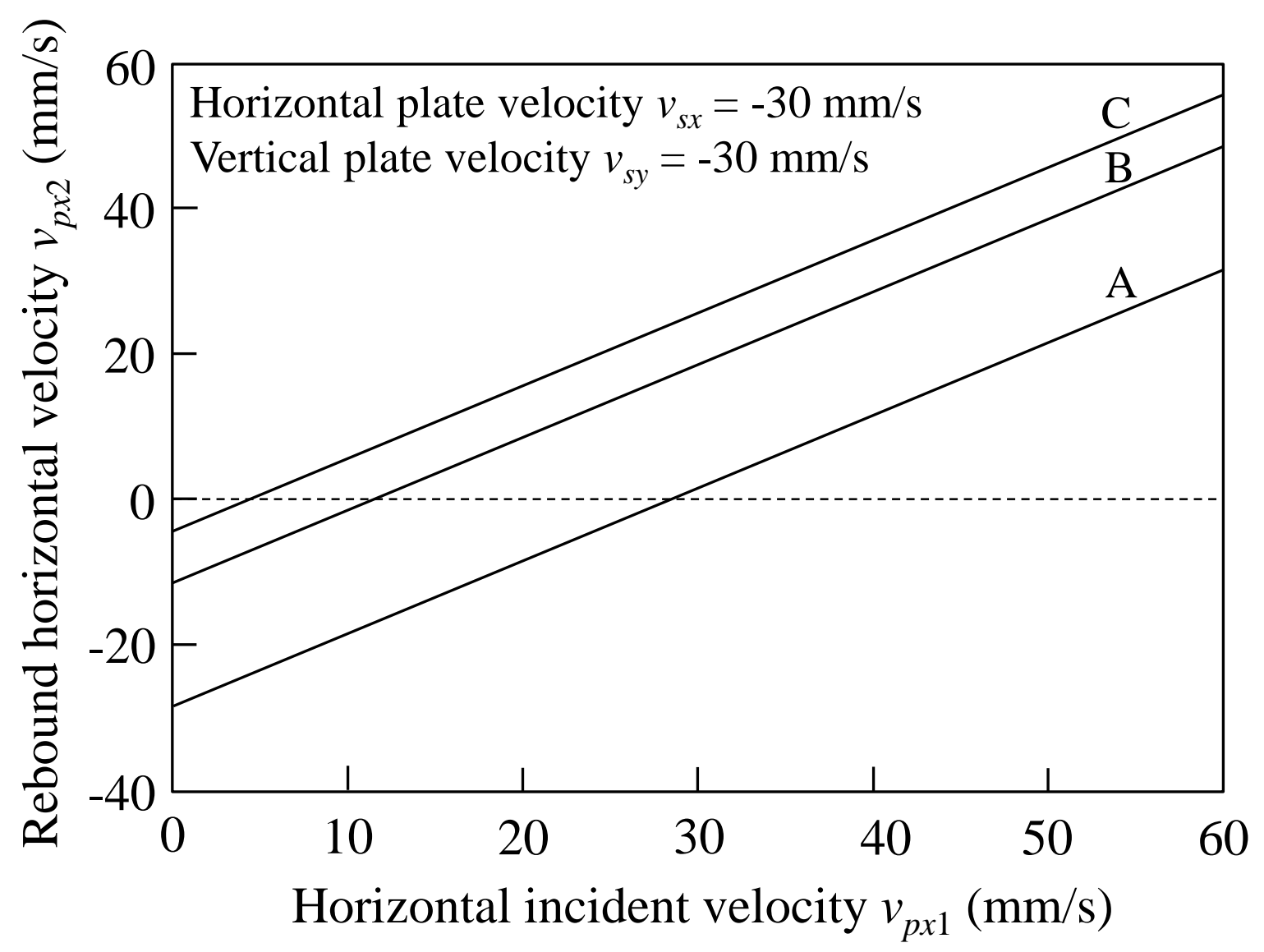


Fig. 8.

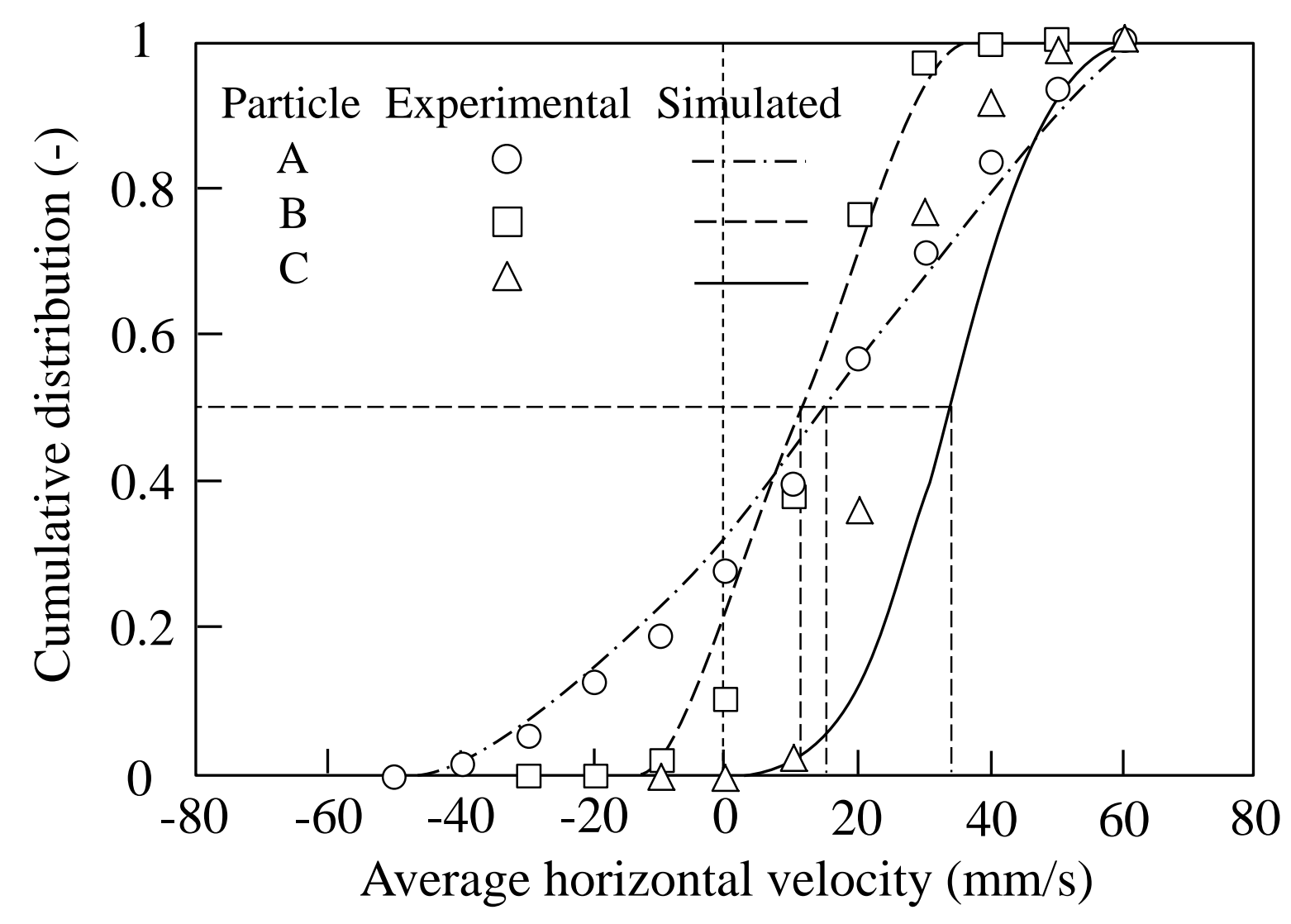

